\title{
Fundamental laws and principles in geoinformation science
}

\author{
Mikko Vastaranta ${ }^{1}$, Ninni Saarinen ${ }^{1,2}$, Tuomas Yrttimaa ${ }^{1}$, Timo Tokola ${ }^{1}$ \\ ${ }^{1}$ School of Forest Sciences, University of Eastern Finland, Joensuu, 80101, Finland \\ ${ }^{2}$ Department of Forest Sciences, University of Helsinki, Helsinki, 00014, Finland; \\ mikko.vastaranta@uef.fi, ninni.saarinen@helsinki.fi, tuomas.yrttimaa@uef.fi, timo.tokola@uef.fi
}

\begin{abstract}
Scientific laws are empirical statements, based on repeated experiments or observations, that describe or predict a range of natural phenomena. There are scientific laws and law-like statements also in the field of geoinformation sciences. Based on the Tobler's first law of geography, "everything is related to everything else, but near things are more related than distant things". This first law is the foundation of the fundamental concepts of spatial dependence and spatial autocorrelation. Anselin's second principle of spatial heterogeneity argues that expectations vary across the Earth's surface, with the important consequence that the results of any analysis depend explicitly on the bounds of the analysis. There are also some other law-like statements that are covered here. Geoinformation science is built upon theoretical foundations that have been developed in other fields such as geography, ecology, statistics, demography, operations research, sociology, mathematics, and computer science.
\end{abstract}

\section{Scientific theories, laws and principles}

We will first clarify what is the difference between scientific theory, law and principle. A scientific theory is an explanation of an aspect of the natural world that can be repeatedly tested and verified in accordance with the scientific method, using accepted protocols of observation, measurement, and evaluation of results. Where possible, scientific theories are tested under controlled conditions with an experiment [1]. Scientific laws or laws of science are also empirical statements, based on repeated experiments or observations, but they describe or predict a range of natural phenomena [2]. So, here is a clear difference between a scientific theory and a scientific law. In other words, scientific theories are related to one specific phenomenon whereas scientific laws can explain a range of phenomena. Scientific laws and theories are both developed from (empirical) data and are without an exception directly or indirectly based on empirical 
evidence. It is generally understood that scientific laws are discovered rather than invented [3]. Scientific laws and theories are also typically uniform in space and time. A scientific principle, on the other hand, is defined here as a proposition that serves as the foundation for something or in other words a scientific principle explains how something happens or works (e.g. the principle of quantum theory). The difference between a scientific theory and a scientific principle here is that a scientific principle does not have to be empirically tested as those can be deductively reasoned. However, this is a rather thin line here.

There are few statements that have been proposed as scientific laws in the field of geoinformation sciences, they are considered here as such and presented in the following sections. Some of these law-like statements are based on empirical observations and others are dealing with the geographic form rather than a process. Laws based on empirical observations to be presented here have already been observed to be generally true. If some deviation from these laws are to be detected should it scientifically be very interesting. According to Goodchild [4], it has been questioned that can there even be laws or law-like statements in the social sciences including geoinformation science. If a single counterexample could defeat a law then it might be impossible. However, development of laws and law-like statements allow teaching to be structured based on the first principles and provide the basis for predicting performance as well as making theoretically sound design choices.

\section{Scientific laws and principles related to geoinformation science}

\section{1 Tobler's first law of geography}

"Everything is related to everything else, but near things are more related than distant things." [5]. This Tobler's first law is the foundation of the fundamental concepts of spatial dependence and spatial autocorrelation. Spatial dependence describes the widely observed tendency for the variance of spatial data to increase with distance. In other words, "nearby things are more similar than distant things". All methods used for representing geographic phenomena in geoinformation systems (GISs) are dependent, to some extent, on the validity of this scientific law. For example, interpolation of soil class or air temperature would be impossible if this law would not be valid (Figure 1). There would be no value in representing topography with isolines if elevation did not vary smoothly. It can be said that GIS and GIS technologies are conceptually based on Tobler's First Law of Geography. This law is also rather similar to the First Law of Cognitive Geography [6] which is formulated as "People think that closer things are more similar". 


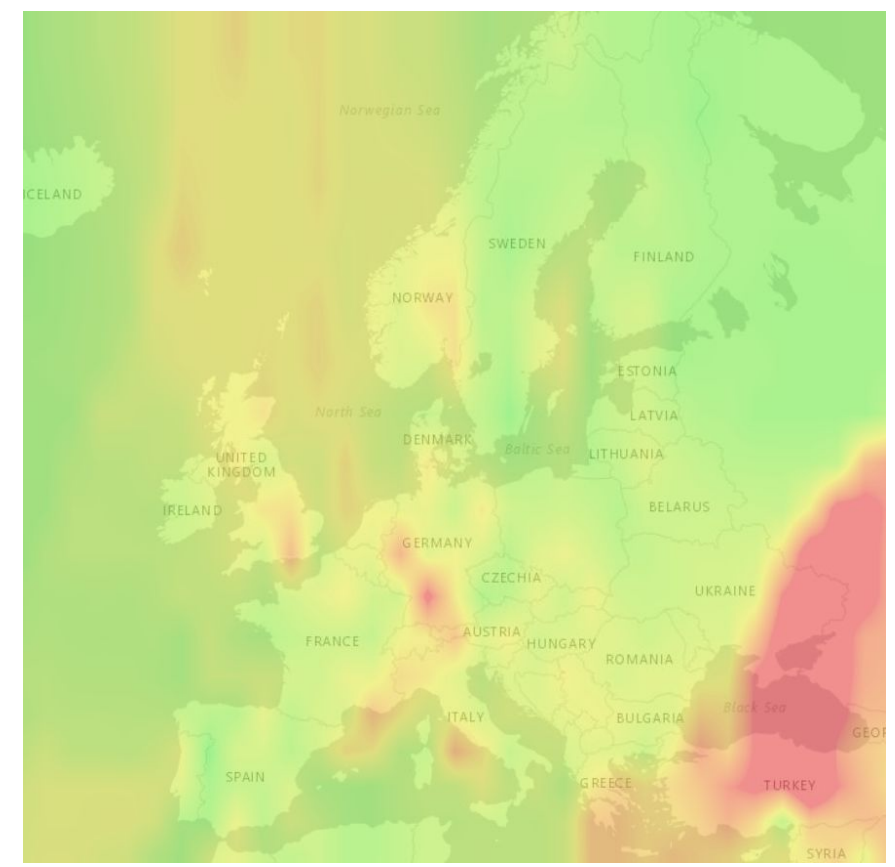

Figure 1. Interpolation of air pollution maps based on observed data (points, weather stations) is possible, because "nearby things are more similar than distant things".

\section{2 Anselin's second principle of spatial heterogeneity}

The Anselin's second principle of spatial heterogeneity [7] argues that expectations vary across the Earth's surface, with the important consequence that the results of any analysis depend explicitly on the bounds of the analysis. One of the practical implications is that a state is not a sample of the nation - a country is not a sample of the world. Thus, there is no "average place" on the Earth's surface - it is impossible. For example, forest conditions change from place to place due to the tendency for the Earth's surface to exhibit spatial non-stationarity. To clarify, spatial nonstationarity is a condition in which a simple "global" model cannot explain the relationships between some sets of variables. For example, you are trying to model the relationship between two variables, diameter at breast height ( $\mathrm{dbh}$ ) and height of the trees, in a given area of interest. Using a global linear regression, you find that there is a positive correlation between the dbh and height. However, you can detect differences in this relation between stands due to tree density and site characteristics. Thus, the relationship you are modeling is non-stationary throughout space. The implication that there is no "average place" on the Earth's surface can be demonstrated with national forest inventory (NFI) as well. With the NFI data, you can easily derive forest structural attributes for an average forest. However, due to this scientific principle, that kind of forest - although it should just be "an average forest" - cannot be found in the real world. Just by thinking about the required mixture of the species and values for all the 
forest structural attributes, it is easily seen as an impossible task. Various techniques developed over the past decades for local spatial analysis, such as geographically weighted regression (or also mixed-effect modelling which however, does not have to use geospatial data) are based on this principle, since they attempt to summarize what is true locally, rather than what is true globally [4].

\section{3 Other law-like statements and theoretical foundations}

There are also some other suggestions for law-like statements related to geoinformation science. First one is the fractal principle [4]. It can be expressed as "the closer you look, the more you see". This means that all geographic phenomena reveal more detail with finer spatial resolution, at predictable rates. This principle has practical implications for many GIS analyses. The classic example is that length is always a function of spatial resolution (Figure 2). If you think about lengths of national boundaries, depending on the used resolution, you always get a different answer. Somehow, this principle is often forgotten and the scale for the analyses is selected implicitly and thought that the "right" answers are obtained if analyses are carried out properly. Fractal principle should always be taken into account in topographical analysis and spatial resolution (i.e. scale dimension) should always be explicit in GIS analysis.
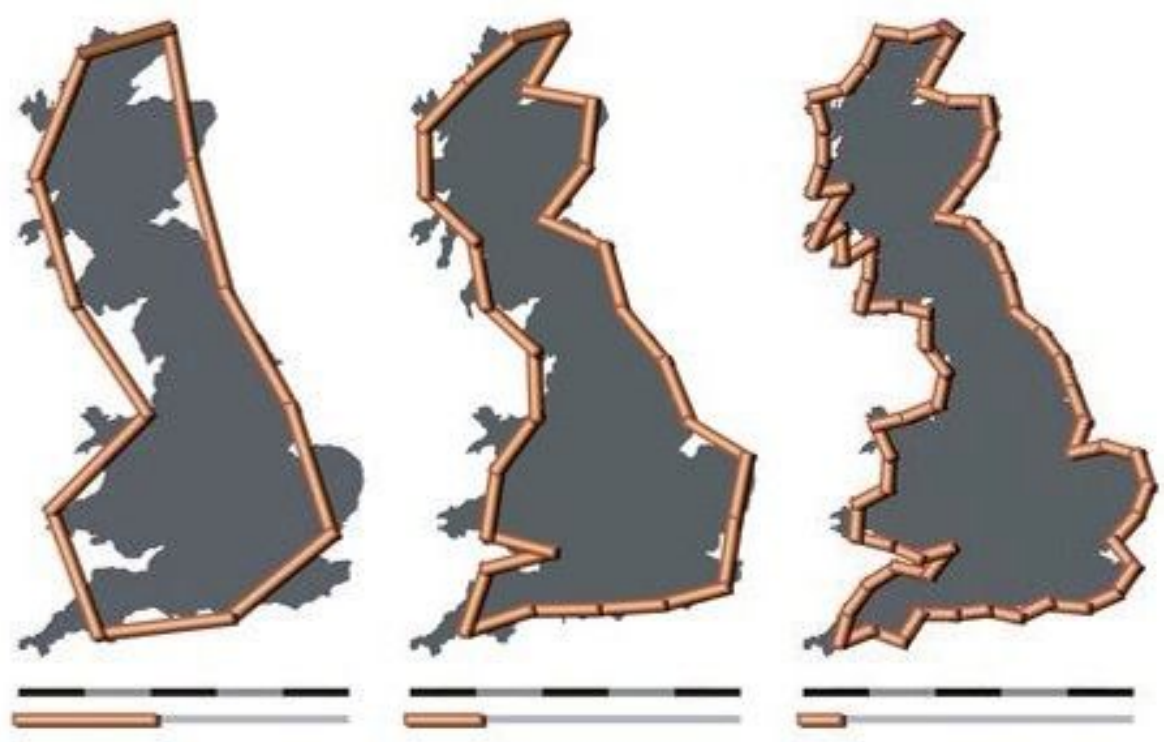

Figure 2. Due to fractal principle, length is a function of spatial resolution. For example, the coastline of the United Kingdom as measured with measuring rods of $200 \mathrm{~km}, 100 \mathrm{~km}$ and $50 \mathrm{~km}$ in length. The resulting coastline is about $2350 \mathrm{~km}, 2775 \mathrm{~km}$ and $3425 \mathrm{~km}$; the shorter the scale, the longer the measured length of the coast. This problem is also known as coastline paradox. Image by Alexandre Van de 
Sandeby is licensed under CC BY-SA 3.0.

Then there is the uncertainty principle [4]. It means that it is impossible to measure location or to describe geographic phenomena exactly. As we are limited to using points, lines, polygons and rasters, we should keep in mind that no representation of the Earth's surface or an area can be complete. Furthermore, there is a principle dealing with the geographic form, relating to approaches how geographic variation can be conceptualized. There are only two ways to do it, either as discrete, countable objects or as a collection of continuous fields, functions of location [4]. If you think about the tree density and height variation. Tree density variation can be presented simply by using points. When points are close to each other, tree density is higher. Tree height variation can be illustrated using raster, in which each raster cell value represents height above the ground. Of course, tree density variation can be represented using density surface raster and tree height variation by using different themes for each height class of the points. This principle is related, again, to the principle of uncertainty and limitation of describing the real world using vector and raster data. Goodchild [4] calls this principle "Objects and Fields".

Other theories related to geoinformation sciences include theories about spatial relations, such as topology. Geospatial topology studies the rules concerning the relationships between points, lines, and polygons. For example, where two polygons represent adjacent countries, typical topological rules would require that the countries share a common boundary with no gaps and no overlaps. Temporal topology is, respectively, the expression of relationships between events in time. Furthermore, it is not often considered how language defines the spatial order of objects through prepositions, for example. There are theories concerning the relations of the partial and total order of spatial objects as described by prepositions such as in front of, behind, above, and below (e.g. [8]). Geoinformation science is also an applied science, which means that many of its theoretical foundations are originally developed in geography, ecology, statistics, demography, operations research, sociology, mathematics, computer science or in other fields.

Author Contributions: Writing—original draft preparation, M.V.; writing—review and editing, all the authors.

\section{References}

1. Winther, R.G. The Structure of Scientific Theories. 2015.

2. Armstrong, D.M. Laws of nature as relations between universals. What is a Law of Nature? 2016, 71-102.

3. McComas, W.F. The Language of Science Education: An Expanded Glossary of Key Terms and Concepts in Science Teaching and Learning; Springer Science \& Business Media, 2013; ISBN 9789462094970.

4. Goodchild, M.F. The fundamental laws of GIScience 2003.

5. Tobler, W.R. A Computer Movie Simulating Urban Growth in the Detroit Region. Economic 
Geography 1970, 46, 234.

6. Montello, D.R.; Fabrikant, S.I.; Ruocco, M.; Middleton, R.S. Testing the First Law of Cognitive Geography on Point-Display Spatializations. Spatial Information Theory. Foundations of Geographic Information Science 2003, 316-331.

7. Anselin, L. What is Special about Spatial Data?: Alternative Perspectives on Spatial Data Analysis; 1989;.

8. Platonov, G.; Schubert, L. Computational Models for Spatial Prepositions. Proceedings of the First International Workshop on Spatial Language Understanding 2018. 